\title{
Glycyrrhizic acid prevents enteritis through reduction of NF-кB p65 and p38MAPK expression in rat
}

\author{
YI-MING WANG and GUO-QIANG DU \\ Department of Pediatric Surgery, Linyi People's Hospital, Linyi, Shandong 276003, P.R. China \\ Received May 6, 2015; Accepted January 7, 2016
}

DOI: $10.3892 / \mathrm{mmr} .2016 .4981$

\begin{abstract}
Glycyrrhizic acid has a variety of biological properties, including a protective function in the liver, and anti-inflammatory, anti-ulcer, anti-anaphylaxis, anti-oxidant, immunoregulatory, antiviral and anticancer activities. The efficacy of glycyrrhizic acid can be increased when combined with other medicines. In the present study, the potential protective effects of glycyrrhizic acid against enteritis in rats, and its role in regulating anti-inflammation, anti-oxidation, angiogenic and apoptotic mechanisms were investigated using enzyme-linked immunosorbent and bicinchoninic acid assays, and reverse transcription-quantitative polymerase chain reaction and western blotting analyses. Adult male Sprague-Dawley rats were injected with $20 \mathrm{mg} / \mathrm{kg}$ methotrexate (MTX) to establish enteritis. Additionally, rats with MTX-induced enteritis were peritoneally injected with $200 \mathrm{mg}$ glycyrrhizic acid for 9 weeks. The current study demonstrated that glycyrrhizic acid could alleviate MTX-induced increases of tumor necrosis factor- $\alpha$, interleukin (IL)- $1 \beta$ and IL-6 levels, and raise IL-10 levels, in rats with enteritis. Treatment with glycyrrhizic acid significantly reduced D-lactate and intercellular adhesion molecule-1 gene expression $(\mathrm{P}<0.01)$, but did not inhibit diamine oxidase activity in MTX-induced enteritis. Pretreatment with glycyrrhizic acid significantly suppressed the promotion of $\mathrm{p} 38$ mitogen-activated protein kinase (p38MAPK), nuclear factor- $\kappa \mathrm{B}$ p65 (NF- $\kappa \mathrm{B}$ p65) protein expression, interferon $\gamma$ protein concentration, and caspase- 3 and cycloxygenase- 2 activity in MTX-induced enteritis $(\mathrm{P}<0.01)$. The findings of the current study suggest that glycyrrhizic acid may prevent enteritis by reducing NF- $\mathrm{\kappa B}$ p65 and p38MAPK expression levels, which may inform future therapeutic strategies for the treatment of enteritis.
\end{abstract}

Correspondence to: Mr. Guo-Qiang Du, Department of Pediatric Surgery, Linyi People's Hospital, 27 Jiefang Road, Linyi, Shandong 276003, P.R. China

E-mail: dudoctordgq@163.com

Key words: glycyrrhizic acid, enteritis, nuclear factor- $\kappa \mathrm{B}$ p65, p38 mitogen-activated protein kinase

\section{Introduction}

The intestinal mucosa is the major location of nutrient digestion and absorption, as well as the primary gateway for invasion by pathogenic microorganisms and their toxins (1). Under physiological conditions, extra-intestinal tissues and organs are effectively protected from pathogenic microorganisms and their toxins by the intestinal mucosa barrier (IMB) (2). The aforementioned mechanism involves the IMB, intestinal mucosa immune barrier, micropopulation barrier, chemical barrier, slime layer and aqueous layer (3). As a result, when the IMB is damaged, microorganisms and their toxins can move through the IMB, and enter the portal vein and lymphatic system, leading to bacterial translocation, potentially causing systemic inflammatory response syndrome and multiple organ dysfunction syndrome (4).

Dysfunction of the IMB is closely associated with diseases of the digestive system. Numerous factors, including intestinal infection, inflammation and mechanical damage, can induce abnormal IMB function (5). IMB dysfunction increases intestinal mucosa permeability, causing bacteria and antigens to be translocated from the enteric cavity to the lamina propria mucosae, resulting in the activation of immune cells and an abnormal mucosal immune response (6). Inflammatory bowel diseases, such as enteritis, can also damage the IMB by increasing the release of inflammatory cytokines, further exacerbating the abnormal mucosal immune response (7). It is widely understood that IMB dysfunction causes the molecular pathogenesis of enteritis $(7,8)$.

Glycyrrhiza, a group of perennial leguminous grasses, have been used in traditional medicine in Asian countries, including China, India and Japan, for thousands of years, and are now widely used in Europe and the Middle East (9). Glycyrrhizic acid is water-soluble compound, consisting of two molecules of glucuronic acid and one molecule of glycyrrhetinic acid (10). Glycyrrhizic acid, which exhibits a series of effects, such as liver protection and membrane stabilization, is one of the major constituents of Glycyrrhiza root extract. Its biological effects include anti-inflammatory, anti-ulcer, anti-anaphylaxis, anti-oxidant, immunoregulatory, membrane stabilization, antiviral and anticancer activities, as well as liver protective properties $(9,10)$. The pentacyclic triterpene structure in glycyrrhetinic acid gives glycyrrhizic acid a similar structure to glucocorticoids and, as such, similar anti-inflammatory effects (11). The anti-inflammatory effect of glycyrrhizic 
acid does not cause severe adverse reactions, therefore, it is widely used in the treatment of acute and chronic hepatitis, bronchitis, acquired immune deficiency syndrome and other diseases $(12,13)$.

Nuclear factor (NF)- $\kappa$ B p65 is a common transcription factor which is activated by inflammatory cytokines, growth factor or chemokines in order to regulate transcription (14). Following stimulation by an inflammatory cytokine or various cytokines, a trimer of NF- $\kappa \mathrm{B}$ and $\mathrm{I} \kappa \mathrm{Ba}$ dissociates in the cytoplasm, and phosphorylation of I $\kappa \mathrm{Ba}$ facilitates nuclear localization to induce $\mathrm{NF}-\kappa \mathrm{B}$ expression, which is a classical NF- $\mathrm{NB}$ pathway (15). Following nuclear entry, $\mathrm{NF}-\kappa \mathrm{B}$ can regulate gene expression. Positive and negative feedback loops and subsequent inflammatory reactions further affect the inflammatory reaction of the body (16).

Mitogen activated protein kinase (MAPK) signal transduction pathways are associated with cell proliferation, differentiation, cell apoptosis and angiogenesis (17). In particular, the p38MAPK signal transduction pathway regulates stress responses, such as inflammation and cell apoptosis (18). Following the activation of MAPK pathways by lipopolysaccharides and other factors, numerous inflammatory mediators are generated via complex signal conduction pathways, which promote inflammation. p38MAPK can be activated by various stimulus along diversified transduction pathways which activate numerous transcription factors and mediate a range of biological effects (19).

The present study aimed to investigate the mechanism by which glycyrrhizic acid prevents enteritis in rats and whether its effects are mediated via reduction of $\mathrm{NF}-\kappa \mathrm{B}$ p65 and p38MAPK expression levels.

\section{Materials and methods}

Chemicals. Methotrexate (MTX) was purchased from Shanghai Zhongxi Sunve Pharmaceutical Co., Ltd. (Shanghai, China). Tumor necrosis factor- $\alpha$ (TNF- $\alpha$; MTA00), interleukin-1 $\beta$ (IL-1 $\beta$; DLB50), IL-6 (D6050), IL-10 (D1000B), cycloxygenase-2 (COX-2; KCB4198) and interferon- $\gamma$ (IFN- $\gamma$; RIF00) enzyme-linked immunosorbent assay (ELISA) kits were purchased from R\&D Systems Europe, Ltd. (Abingdon, UK). Bicinchoninic acid (BCA) assay kit (P0010S) was purchased from Beyotime Institute of Biotechnology (Jiangsu, China).

MTX-induced enteritis rat model and grouping. A total of 24 adult male Sprague-Dawley rats, aged 6-7 weeks and weighing 220-250 g, were obtained from the Animal Resource Center of the Linyi People's Hospital (Linyi, China). Rats were housed at an ambient temperature of $22 \pm 1^{\circ} \mathrm{C}$, with a $12-\mathrm{h}$ light-dark cycle, and free access to food and water. The current study was conducted in accordance with the recommendations of the Guide for the Care and Use of Laboratory Animals by the National Institutes of Health (20) and the protocol was approved by the Ethics Committee of Linyi People's Hospital. The experimental rats were randomly allocated into three equal groups: Control, MTX and glycyrrhizic acid groups ( $n=8$ per group). In the control group, normal rats were peritoneally injected with $1 \mathrm{ml}$ normal saline. In the MTX and glycyrrhizic acid groups, enteritis was induced in rats by injection with $20 \mathrm{mg} / \mathrm{kg}$ MTX. In the MTX group, MTX-induced rats were peritoneally injected with $1 \mathrm{ml}$ normal saline. In the glycyrrhizic acid group, MTX-induced rats were peritoneally injected with $200 \mathrm{mg}$ glycyrrhizic acid for 9 weeks (21). After 9 weeks, rats was sacrificed via decollation and blood samples and intestinal tissue samples were subsequently harvested. Blood samples were centrifuged at $13,200 \mathrm{x} \mathrm{g}$ for $10 \mathrm{~min}$ at $4^{\circ} \mathrm{C}$ to obtain the serum. Serum and intestinal tissue samples were stored at $-80^{\circ} \mathrm{C}$ prior to analysis.

Collection of rat blood samples and measurement of inflammatory mediators by ELISA. Peripheral blood was collected after glycyrrhizic acid treatment and centrifuged at 13,200 x g for $10 \mathrm{~min}$ at $4^{\circ} \mathrm{C}$. The supernatant was collected, and the levels of TNF- $\alpha$, IL-1 $\beta$, IL-6, IL-10, IFN- $\gamma$ and COX-2 were measured using the described ELISA kits, according to the manufacturer's protocol.

Measurement of plasma D-lactate concentration and diamine oxidase (DAO) activity. Peripheral blood supernatant was obtained as described for the ELISAs. The D-lactate concentration levels and DAO activity were measured using spectrophotometry (Multiskan MS plate reader; Thermo Fisher Scientific, Inc., Waltham, MA, USA), as previously described $(22,23)$.

Reverse transcription-quantitative polymerase chain reaction (RT-qPCR) of intercellular adhesion molecule-1 (ICAM-1). Total RNA was extracted from rat intestinal tissue samples using TRIzol reagent (Invitrogen; Thermo Fisher Scientific, Inc.), according to the manufacturer's protocol. The extracted RNA $(1 \mu \mathrm{g})$ was reverse transcribed into cDNA using a SuperScript III First-Strand Synthesis system (Invitrogen; Thermo Fisher Scientific, Inc.). qPCR was performed on a Gene Amp 2400 PCR system (PerkinElmer, Inc., Waltham, MA, USA) using a Premix Ex Taq kit (Takara Bio, Inc., Kyoto, Japan), according to the manufacturer's protocol as follows: $94^{\circ} \mathrm{C}$ for $5 \mathrm{~min}$ followed by 40 cycles of $95^{\circ} \mathrm{C}$ for $30 \mathrm{sec}, 60^{\circ} \mathrm{C}$ for $45 \mathrm{sec}$ and $72^{\circ} \mathrm{C}$ for $30 \mathrm{sec}$, and $72^{\circ} \mathrm{C}$ for $10 \mathrm{~min}$. Primer sequences were as follows: ICAM-1 forward 5'-AACGACGCTTCTTTT GCTC-3' and reverse 5'-CTCTGGCGGTAATAGGTGTAA-3'; and GAPDH (reference) forward 5'-CGTGTTCCTACCCCC AATGT-3' and reverse 5'-TGTCATACTTGGCAGGTTTCT-3'. Relative expression levels were normalized against GAPDH, using the $2^{-\Delta \Delta \mathrm{Cq}}$ method (24).

Western blotting. Intestinal tissue samples were collected and incubated with $100 \mu \mathrm{l}$ tissue lysis buffer (Beyotime Institute of Biotechnology) for $30 \mathrm{~min}$ on ice. Homogenates were centrifuged at $13,200 \times \mathrm{g}$ for $10 \mathrm{~min}$ at $4^{\circ} \mathrm{C}$, the supernatant was collected and the protein concentration measured using a the BCA kit, according to the manufacturer's protocol. Equal concentrations of protein sample were separated by running on $10 \%$ SDS-PAGE gels (Beijing Solarbio Science \& Technology Co., Ltd., Beijing, China) at $100 \mathrm{~V}$ for $75 \mathrm{~min}$ and transferred onto polyvinylidene difluoride membranes (0.22 mm; Bio-Rad Laboratories, Inc., Munich, Germany). The membrane was incubated with anti-NF- $\kappa$ B-p 65 (sc-372; 1,2,000), rabbit polyclonal anti-phosphorylated (p)-p38MAPK (sc-101758), anti-p38MAPK (sc-728), rabbit polyclonal anti-iNOS (sc-651; all 1:1,000) or $\beta$-actin $(1: 2,000$; sc-130656; all Santa Cruz Biotechnology, Inc., Dallas, TX, 
USA) primary antibodies overnight at $4^{\circ} \mathrm{C}$. Membranes were then incubated with the appropriate horseradish peroxidase-conjugated secondary antibody (sc-2370; 1:2,000; Santa Cruz Biotechnology, Inc.) and enhanced chemiluminescence kit (GE Healthcare Life Sciences, Chalfont, UK). The relative protein expression was determined using a Gel Dox 1000 fluorescent image analysis system (Bio-Rad Laboratories, Inc.).

Caspase-3 activity. Protein was extracted from intestinal tissue samples, as described for western blotting, and quantified using the BCA assay kit, according to the manufacturer's protocol. Equal concentrations of protein were incubated with Ac-DEVD-pNA (Beyotime Institute of Biotechnology) at $37^{\circ} \mathrm{C}$ for $2 \mathrm{~h}$ in the dark, as previously described (25), and the absorbance at $405 \mathrm{~nm}$ was measured using a Varioskan Flash spectral scanning multimode reader.

Statistical analysis. Data from the individual groups were presented as the mean \pm standard deviation and were analyzed using analysis of variance followed by Tukey-Kramer multiple comparisons test. Statistical analyses were performed using SPSS 17.0 software (SPSS, Inc., Chicago, IL $<$ USA). $\mathrm{P}<0.05$ was considered to indicate a statistically significant difference.

\section{Results}

Glycyrrhizic acid alters the expression of inflammatory factors in rat enteritis. The chemical structure of glycyrrhizic acid (95.0\%; Sigma-Aldrich, St. Louis, MO, USA) is presented in Fig. 1. The current study examined the effect of glycyrrhizic acid on the levels of several inflammatory factors in a rat model of enteritis. The protein concentration levels of TNF- $\alpha$, IL-1 $\beta$ and IL- 6 were significantly increased in rats with MTX-induced enteritis compared with the control group $(\mathrm{P}<0.01$; Fig. 2A-C). Pretreatment with glycyrrhizic acid significantly alleviated the increase of TNF- $\alpha$, IL-1 $\beta$ and IL-6 resulting from MTX-induced enteritis $(\mathrm{P}<0.01$; Fig. 2A-C). Conversely, IL-10 levels were significantly reduced in rats with MTX-induced enteritis compared with the control group $(\mathrm{P}<0.01$; Fig. 2D), and glycyrrhizic acid treatment significantly raised the IL-10 levels compared with the MTX group $(\mathrm{P}<0.01$; Fig. 2D).

D-lactate concentration levels and DAO activity are increased by glycyrrhizic acid in rat enteritis. The current study probed whether glycyrrhizic acid prevents D-lactate and DAO changes that occur in rat enteritis. The D-lactate concentration and DAO activity were significantly increased in MTX-induced enteritis compared with control group $(\mathrm{P}<0.01$; Fig. 3$)$. Furthermore, treatment with glycyrrhizic acid significantly reduced the D-lactate concentration levels compared with the MTX group $(\mathrm{P}<0.01)$, however, glycyrrhizic acid did not inhibit the increased DAO activity in MTX-induced enteritis (Fig. 3B).

ICAM-1 expression is reduced by glycyrrhizic acid in rat enteritis. As presented in Fig. 4, ICAM-1 mRNA expression was significantly higher in MTX-induced enteritis compared with expression in control rats $(\mathrm{P}<0.01)$. However, administration of

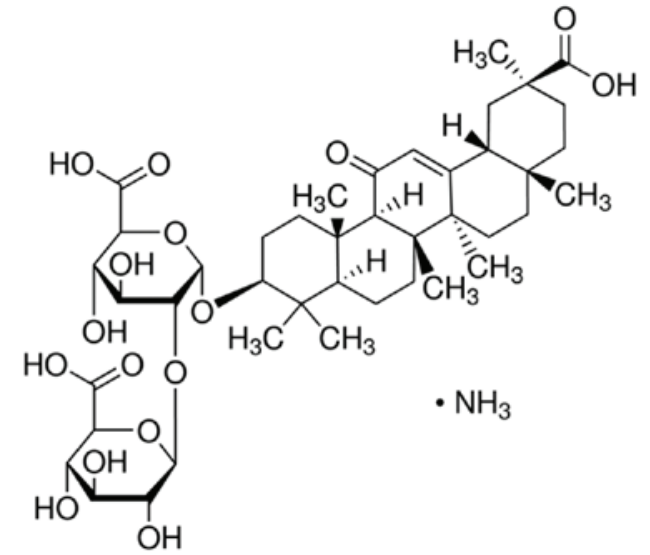

Figure 1. Chemical structure of glycyrrhizic acid.

glycyrrhizic acid significantly reduced the ICAM-1 gene expression compared with MTX-induced enteritis rats ( $\mathrm{P}<0.01$; Fig. 4).

Increase of $N F-\kappa B-p 65$ protein expression in enteritis is prevented by glycyrrhizic acid in rat enteritis. As shown in Fig. 5, MTX-induced enteritis resulted in significantly increased NF- $\mathrm{B}$-p65 expression compared with the control group $(\mathrm{P}<0.01)$. As expected, glycyrrhizic acid significantly suppressed the MTX-induced increase in NF- $\mathrm{B}-\mathrm{p} 65$ expression $(\mathrm{P}<0.01$; Fig. 5).

Glycyrrhizic acid prevents increase in IFN- $\gamma$ concentration levels in rat enteritis. There was a significant increase IFN- $\gamma$ levels in rats with MTX-induced enteritis compared with the control rats $(\mathrm{P}<0.01$; Fig. 6). As presented in Fig. 6, glycyrrhizic acid treatment prevented the promotion of IFN- $\gamma$ levels observed in the MTX group $(\mathrm{P}<0.01)$.

Glycyrrhizic acid reduces caspase-3 activity in rat enteritis. To understand the underlying mechanism of glycyrrhizic-mediated anti-apoptosis, the present study examined whether glycyrrhizic acid could inhibit caspase-3 activity in an MTX-induced enteritis model. As presented in Fig. 7, rats in the MTX group exhibited significantly increased caspase-3 activity compared with control group $(\mathrm{P}<0.01)$. However, the elevation of caspase-3 activity was significantly inhibited by treatment with glycyrrhizic acid compared with the MTX group $(\mathrm{P}<0.01$; Fig. 7$)$.

Glycyrrhizic acid prevents iNOS protein expression in rat enteritis. To examine the underlying mechanisms of glycyrrhizic-mediated changes in nitric oxide (NO) levels, the current study examined whether glycyrrhizic acid inhibits iNOS protein expression in the MTX-induced enteritis rat model. As presented in Fig. 8, the iNOS protein expression was significantly elevated in MTX-induced enteritis compared with the control group $(\mathrm{P}<0.01)$. However, treatment with glycyrrhizic acid reduced the increase of iNOS protein expression, as compared with the MTX-induced enteritis rats (Fig. 8).

Glycyrrhizic acid inhibits $C O X-2$ activity in rat enteritis. To investigate the mechanism by which the anti-oxidant effects of glycyrrhizic acid are mediated, the present study examined the influence of glycyrrhizic acid on COX-2 activity. Compared 

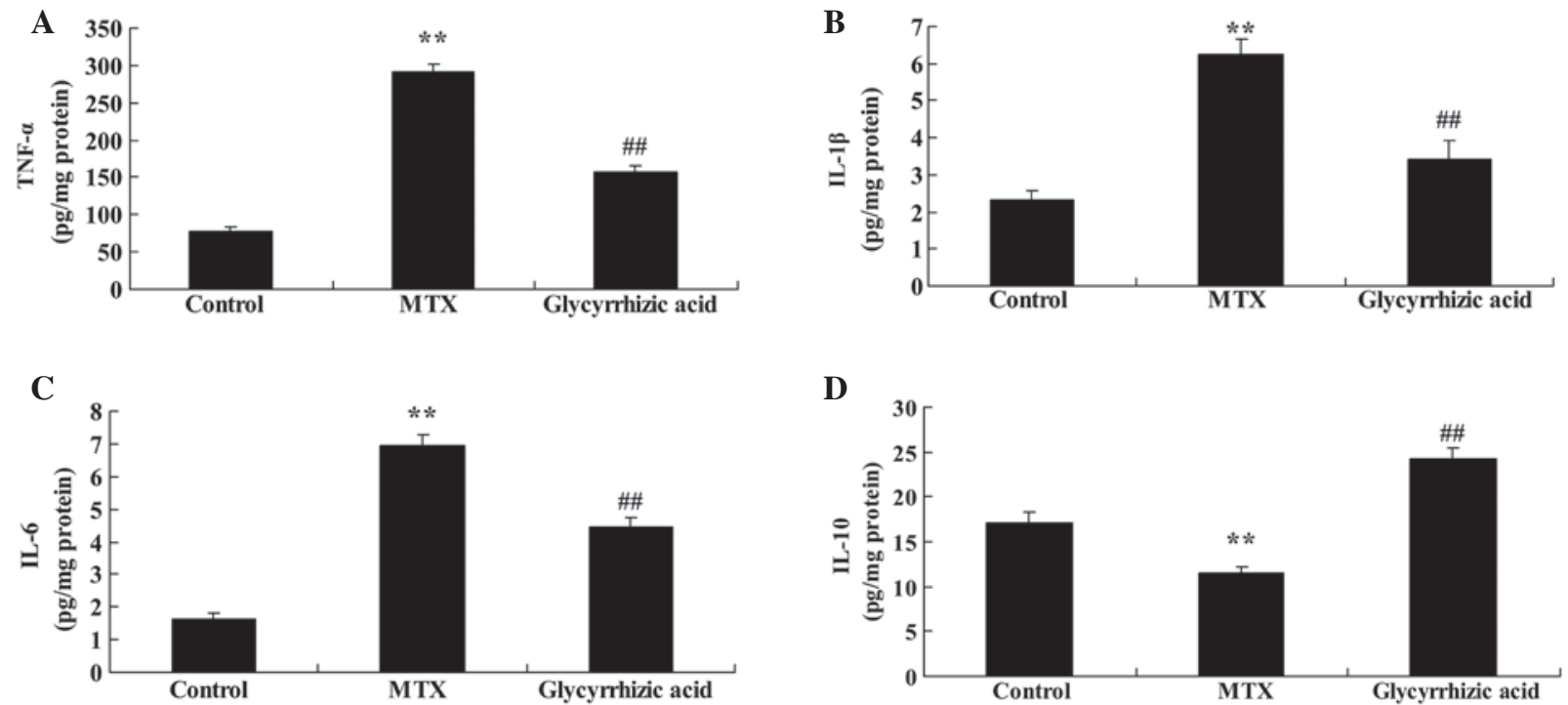

Figure 2. Effect of glycyrrhizic acid on expression of inflammatory factors in enteritis. (A) TNF- $\alpha$, (B) IL-1 $\beta$, (C) IL-6 and (D) IL-10 levels were measured using enzyme-linked immunosorbent assay in control, MTX-induced model and glycyrrhizic acid groups. ${ }^{* *} \mathrm{P}<0.01$ vs. control group; ${ }^{\# \#} \mathrm{P}<0.01$ vs. the MTX group. TNF- $\alpha$, tumor necrosis factor- $\alpha$; IL, interleukin; MTX, methotrexate.

A

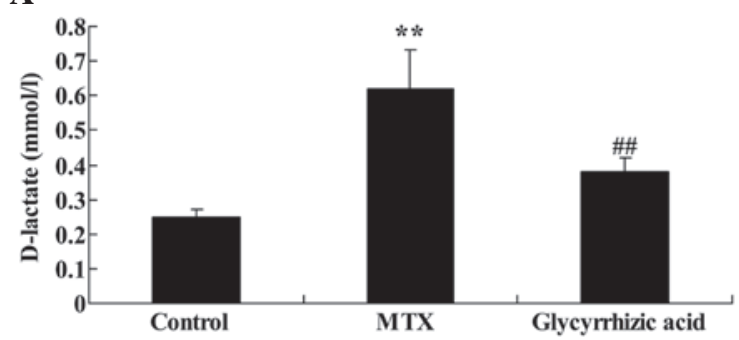

B

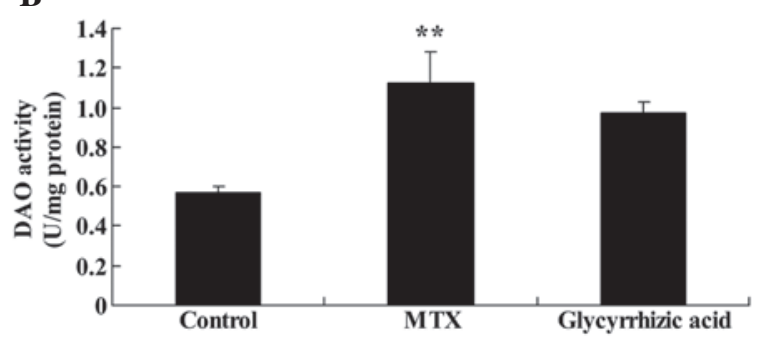

Figure 3. Effect of glycyrrhizic acid on D-lactate and DAO in rat enteritis. (A) D-lactate and (B) DAO were measured in control, MTX-induced model and glycyrrhizic acid groups. ${ }^{* *} \mathrm{P}<0.01 \mathrm{vs}$. control group; ${ }^{\#} \mathrm{P}<0.01$ vs. the MTX group. MTX, methotrexate; DOA, diamine oxidase.

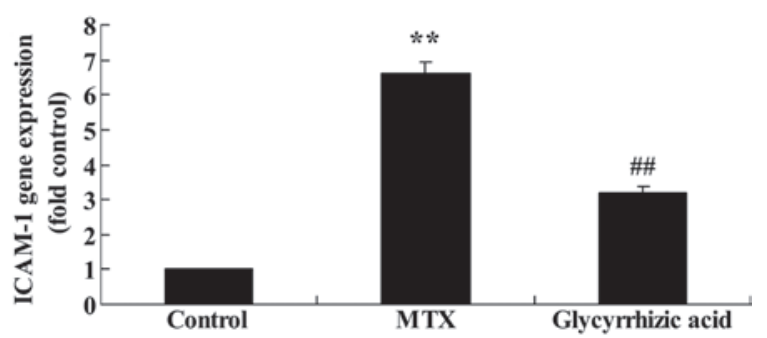

Figure 4. Glycyrrhizic acid prevents ICAM-1 expression in MTX-induced enteritis. ICAM-1 expression was measured in control, MTX-induced model and glycyrrhizic acid groups using reverse transcription-quantiative polymerase chain reaction analysis. ${ }^{* *} \mathrm{P}<0.01$ vs. the control group; ${ }^{\# \prime} \mathrm{P}<0.01$ vs. the MTX group. ICAM-1, intercellular adhesion molecule 1; MTX, methotrexate.

with the control group, the COX-2 activity was significantly increased in rats with MTX-induced enteritis $(\mathrm{P}<0.01$; Fig. 9). This increase was significantly attenuated by glycyrrhizic acid treatment compared with the MTX group ( $\mathrm{P}<0.01$; Fig. 9).

Glycyrrhizic acid prevents p38MAPK expression in rat enteritis. To examine the effects of glycyrrhizic acid on the MAPK signaling pathway, the protein expression levels of total and p-p38MAPK, a key mediator of MAPK signaling, were measured using western blotting. The present study observed that the p38MAPK protein expression was significantly increased in MTX-induced enteritis compared with the control group $(\mathrm{P}<0.01$; Fig. 10). However, treatment with glycyrrhizic acid significantly attenuated the increase in $\mathrm{p} 38 \mathrm{MAPK}$ protein expression observed in the MTX group ( $\mathrm{P}<0.01$; Fig. 10).

\section{Discussion}

IMB dysfunction is closely associated with the paroxysm of enteritis. IMB dysfunction, induced by psychological stress, intestinal infection, mechanical injury and other factors, can increase intestinal mucosa permeability $(1,2)$. Increased mucosa permeability contributes to the translocation of bacteria and antigens from the enteric cavity to the lamina propria mucosae, resulting in activation of immune cells and an abnormal mucosa immune response (26). Additionally, damaging factors are present during the enteritis paroxysm, which can further injure the IMB and aggravate the abnormal mucosa immune response (26). Drugs for the treatment of enteritis can reduce intestinal mucosal inflammation and regulate IMB function (27). IMB function is one of the therapeutic targets in the treatment of enteritis and is an important area of investigation within enteritis pathophysiology research $(26,27)$. 
A

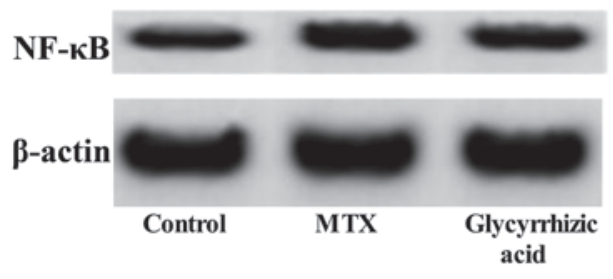

B

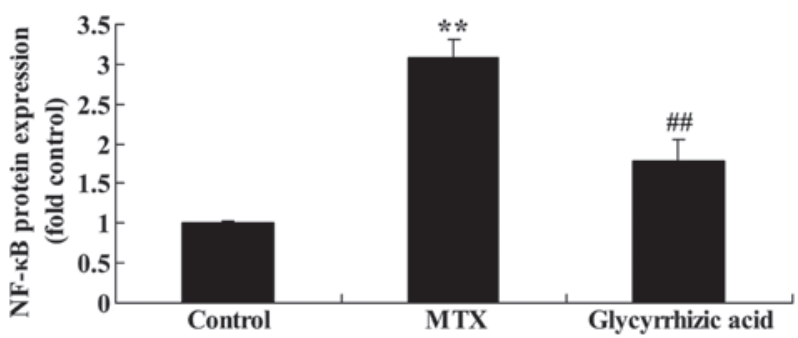

Figure 5. Glycyrrhizic acid prevents NF- $\kappa$ B-p65 expression in rat enteritis. (A) NF- $\kappa$ B-p65 expression was measured by western blotting assays and (B) statistical analysis of NF- $\mathrm{B}$-p65 protein expression was performed using protein from control, MTX-induced model and glycyrrhizic acid groups. ${ }^{*} \mathrm{P}<0.01$ vs. control group; ${ }^{\#} \mathrm{P}<0.01$ vs. the MTX group. NF- $\kappa \mathrm{B}$, nuclear factor- $\kappa \mathrm{B} ; \mathrm{MTX}$, methotrexate.

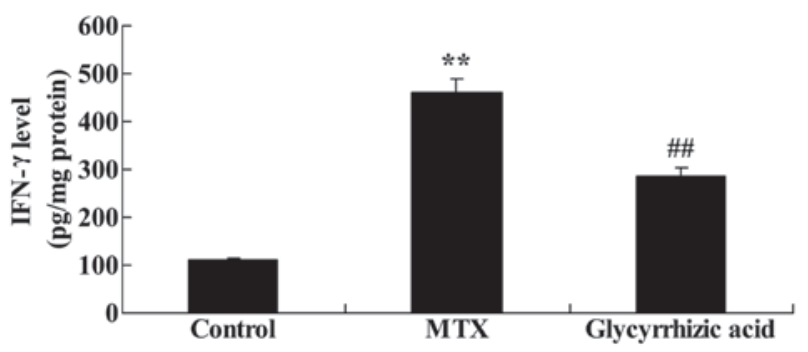

Figure 6. Glycyrrhizic acid reduces IFN- $\gamma$ levels in enteritis rat. IFN- $\gamma$ was measured in control, MTX-induced model and glycyrrhizic acid groups. ${ }^{* *} \mathrm{P}<0.01$ vs. control group; ${ }^{\# \#} \mathrm{P}<0.01$ vs. the MTX group. IFN- $\gamma$, interferon- $\gamma$; MTX, methotrexate.

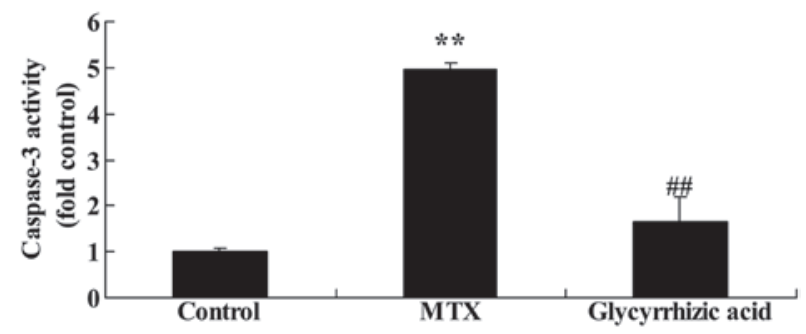

Figure 7. Glycyrrhizic acid prevents caspase-3 activity in enteritis rat. Caspase-3 activity was measured in tissue sample from control, MTX-induced model and glycyrrhizic acid groups. ${ }^{* *} \mathrm{P}<0.01$ vs. control group; ${ }^{\# \#} \mathrm{P}<0.01$ vs. the MTX group. MTX, methotrexate.

The current study observed that pretreatment with glycyrrhizic acid significantly suppressed the concentration levels of various inflammatory mediators that had been increased in MTX-induced enteritis, including TNF- $\alpha$, IL-1 $\beta$, IL-6 and D-lactate levels, whereas, IL-10 levels were reduced in enteritis and increased by glycyrrhizic acid. Bhattacharjee et al (28) demonstrated that glycyrrhizic acid suppresses inflammatory responses and protects against Leishmania parasites within the host. Orazizadeh et al (29) observed that glycyrrhizic acid protects against nanoparticle-induced hepatotoxicity through anti-inflammatory mechanisms, and suggested that glycyrrhizic acid may be a novel candidate for research and development of enteritis therapeutics.

ICAM-1 is expressed on the membrane of endothelial cells, white blood cells (WBC) and enterocytes of intestinal tissues, acting as a receptor for $\beta 2$-integrin present on WBCs. Therefore, ICAM-1 is important in leukocyte movement and aggregation during enteritis (30). Enteritis also induces the expression of adhesion molecules, including ICAM-1 (31). During the acute phase of enteritis, expression of ICAM-1 on the surface of activated vascular endothelial cells is markedly increased, while the mortality of ICAM-1-deficient mice is markedly reduced (30). Lower mortality, and reduced inflammatory cell infiltration and injury of the intestinal tract in ICAM-1-deficient mice indicates that ICAM-1 is important in the development of enteritis (31). The present data demonstrated that administration of glycyrrhizic acid significantly reduces ICAM-1 mRNA expression in MTX-induced enteritis rat. In addition, Wang et al (21) suggested that glycyrrhizic acid attenuates glycative stress in the kidneys of diabetic mice through inhibition of monocyte chemotactic protein-1 and ICAM-1. Together, these results suggest that the anti-inflammatory activity of glycyrrhizic acid during enteritis may be partially mediated through inhibition of ICAM-1.

IFNs are a group of broad-spectrum anti-viral glycoproteins secreted by cells upon attack by viruses. IFN- $\gamma$, which is secreted by lymphocytes, is involved in the expression of histocompatibility antigen and immune adjustment (32). It is understood that the interaction between IFN- $\gamma$ and TNF- $\alpha$ can change the structure of intestinal epithelial cells and the barrier function, leading to increased intestine permeability. Increased expression of IFN- $\gamma$ is commonly observed in enteritis $(4,32)$. In agreement with this, the present study demonstrated that glycyrrhizic acid could reduced MTX-induced NF- $\kappa \mathrm{B}-\mathrm{p} 65$ and IFN- $\gamma$ protein expression in rat enteritis. Feng et al (33) demonstrated that glycyrrhizic acid protects against advanced glycation end-product (AGE)-induced endothelial dysfunction via inhibition of the receptor for $A G E / N F-\kappa B$ signaling pathway. Wu et al (34) reported that glycyrrhizic acid significantly reduced inflammatory via IFN $-\gamma$. This indicates that inhibition of the IFN- $\gamma$ signaling pathway may be associated with the anti-inflammatory effects of glycyrrhizic acid in enteritis.

Enteritis affects intestinal mucosa microcirculation, inflammatory cell infiltration, blood hypercoagulability, microthrombus and inflammatory polyp formation, leading to hypoxia and ischemia of the intestinal mucosa microcirculation, and a severe imbalance of cell factors, including the IL and TNF- $\alpha$ superfamilies, colony stimulating factor, chemokines and growth factors (35). iNOS levels are increased during enteritis and are positively associated with the disease activity index, which reflects the degree of inflammation. iNOS catalyzes the production of NO, which is associated with the pathophysiology 
A

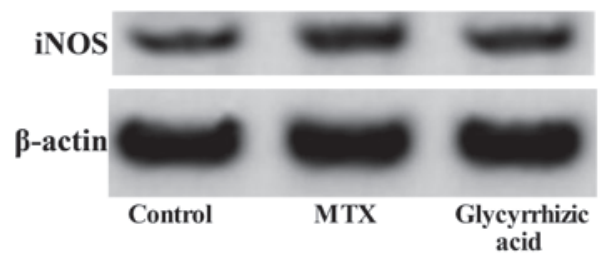

B

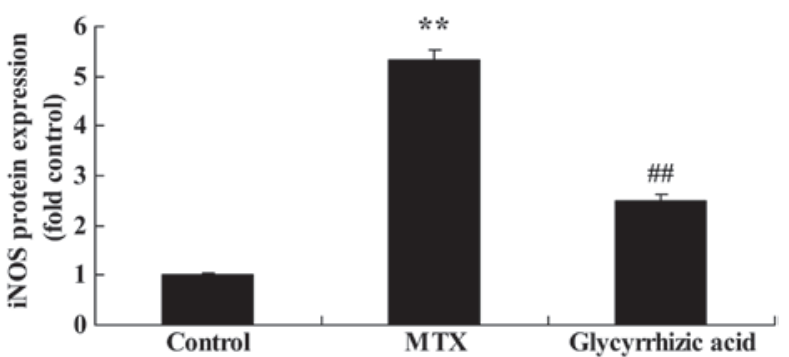

Figure 8. Glycyrrhizic acid prevents iNOS expression in rat enteritis. (A) Western blotting was performed to measure iNOS protein expression and (B) statistically analyzed in samples from control, MTX-induced model and glycyrrhizic acid groups. ${ }^{* *} \mathrm{P}<0.01$ vs. control group; ${ }^{\# \#} \mathrm{P}<0.01$ vs. the MTX group. iNOS, nitric oxide synthase; MTX, methotrexate.

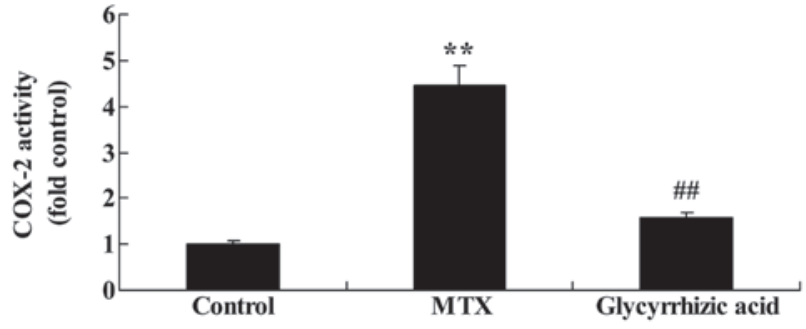

Figure 9. Glycyrrhizic acid prevents COX-2 activity in rat enteritis. COX-2 activity was measured in samples from control, MTX-induced model and glycyrrhizic acid groups. ${ }^{* *} \mathrm{P}<0.01$ vs. control group; ${ }^{\# \#} \mathrm{P}<0.01$ vs. the MTX group. COX-2, cyclooxygenase-2; MTX, methotrexate.

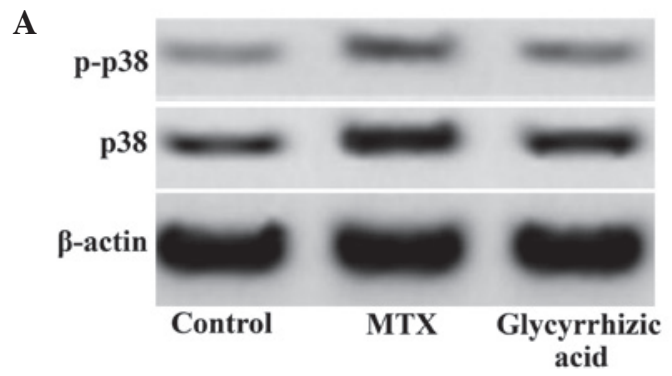

B

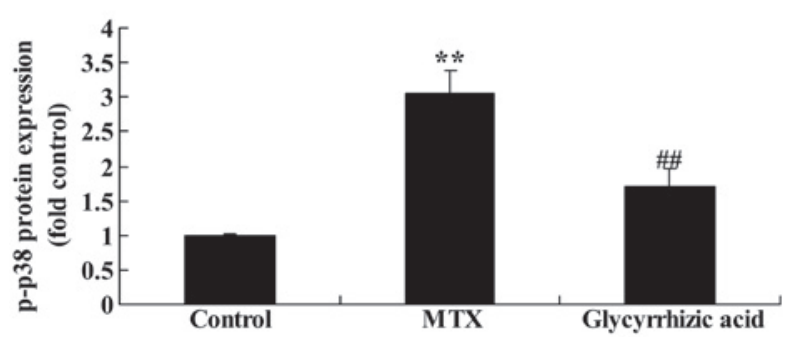

Figure 10. Expression and phosphorylation of $\mathrm{p} 38$ in enteritis rat. (A) p- and total p38 expression were measured in protein samples from control, MTX-induced model and glycyrrhizic acid groups by western blotting and (B) quantified using statistical analysis. ${ }^{* *} \mathrm{P}<0.01$ vs. control group; ${ }^{\# \#} \mathrm{P}<0.01$ vs. the MTX group. p-p38, phosphorylated-p38 mitogen-activated protein kinase; MTX, methotrexate.

of inflammatory diseases and cancer $(5,32)$. The results of the current study demonstrated that glycyrrhizic acid significantly inhibits MTX-induced caspase-3 activity and iNOS expression in enteritis. This suggests that glycyrrhizic acid has anti-apoptotic effects and suppresses iNOS expression during enteritis.
COX-2 is an inducible enzyme that is expressed at low levels in the majority of tissues under normal conditions (36). When induced by pro-infammatory cytokines, several cell types, including endothelial cells, vascular smooth muscle cells, mononuclear macrophages and fibroblasts, rapidly increase the expression of COX-2 to 8 to 10 -fold the normal level (37). Increased COX-2 leads to the production and accumulation of prostaglandin inflammatory factors, promoting inflammatory responses and tissue damage (36). Overexpression of COX-2 promotes cell proliferation, inhibits apoptosis and inhibits the immune response, leading to dysregulation of the balance between proliferation and apoptosis $(36,37)$. The present study demonstrated that glycyrrhizic acid significantly reduces MTX-induced COX-2 activity in enteritis. Bhattacharjee et al (28) also demonstrated that glycyrrhizic acid suppressed COX-2 during L. donovani infection. Additionally, Cherng et al (38) reported that glycyrrhizic acid inhibited NF- $\kappa \mathrm{B}$ and $\mathrm{COX}-2$ expression, prevented DNA damage and facilitated DNA repair.

As an important member of the MAPK signaling pathway, p38MAPK is widely expressed in a variety of tissues (39). Under normal physiological conditions, p38MAPK typically exhibits low activity and is activated upon stimulation by growth factors, lipopolysaccharides and stress (40). Previous research has demonstrated that when cells are stimulated by the aforementioned factors, p38MAPK regulates the inflammatory response via the production of pro-infammatory cytokines, including TNF- $\alpha$, IL-1, IL-6 and IL-8, as well as anti-inflammatory cytokines, such as IL-10 $(40,41)$. As a result, p38MAPK influences the balance between pro- and anti-inflammatory cytokines, thereby influencing processes that cause enteritis (42). The current study demonstrated that glycyrrhizic acid significantly downregulates the protein expression levels of p38MAPK in MTX-induced enteritis. Additionally, several other previous studies demonstrated that glycyrrhizic acid attenuates glycative stress in the kidneys of diabetic mice through suppression of NF- $\kappa \mathrm{B}$ and p-p38MAPK $(21,43)$. These data support the hypothesis that the anti-inflammatory effects of glycyrrhizic acid may be associated with p38MAPK signaling.

In conclusion, the results of the current study demonstrate that glycyrrhizic acid exerts a protective effect during MTX-induced enteritis and may be useful as a therapeutic agent for digestive tract diseases. Furthermore, the protective effect of glycyrrhizic acid is associated with anti-inflammatory and anti-apoptotic pathways, suppression of ICAM-1, 
IFN- $\gamma$, iNOS and COX-2 expression, and downregulation of the p38MAPK pathway. Therefore, glycyrrhizic acid may be a novel therapeutic approach for the treatment of enteritis.

\section{References}

1. Takagi T, Naito Y, Okada H, Takaoka M, Oya-Ito T, Yamada S, Hirai Y, Mizushima K, Yoshida N, Kamada K, et al: Hemopexin is upregulated in rat intestinal mucosa injured by indomethacin. J Gastroenterol Hepatol 27 (Suppl 3): 70-75, 2012.

2. Kim H, Song MJ, Brian H and Choi K: A comparative analysis of ethnomedicinal practices for treating gastrointestinal disorders used by communities living in three national parks (Korea). Evid Based Complement Alternat Med 2014: 108037, 2014.

3. Stoidis CN, Misiakos EP, Patapis P, Fotiadis CI and Spyropoulos BG: Potential benefits of pro- and prebiotics on intestinal mucosal immunity and intestinal barrier in short bowel syndrome. Nutr Res Rev 24: 21-30, 2011.

4. Vandenbroucke RE, Dejonckheere E, Van Hauwermeiren F, Lodens S, De Rycke R, Van Wonterghem E, Staes A, Gevaert K, López-Otin C and Libert C: Matrix metalloproteinase 13 modulates intestinal epithelial barrier integrity in inflammatory diseases by activating TNF. EMBO Mol Med 5: 932-948, 2013.

5. Zhang XP, Zhang J, Song QL and Chen HQ: Mechanism of acute pancreatitis complicated with injury of intestinal mucosa barrier. J Zhejiang Univ Sci B 8: 888-895, 2007.

6. Lin JE, Snook AE, Li P, Stoecker BA, Kim GW, Magee MS, Garcia AV, Valentino MA, Hyslop T, Schulz S and Waldman SA: GUCY2C opposes systemic genotoxic tumorigenesis by regulating AKT-dependent intestinal barrier integrity. PLoS One 7: e31686, 2012.

7. Vetrano S, Ploplis VA, Sala E, Sandoval-Cooper M, Donahue DL, Correale C, Arena V, Spinelli A, Repici A, Malesci A, et al: Unexpected role of anticoagulant protein $\mathrm{C}$ in controlling epithelial barrier integrity and intestinal inflammation. Proc Natl Acad Sci USA 108: 19830-19835, 2011.

8. Talukder JR, Boyd B, Griffin A, Jaima A and Rajendran VM Inflammatory cytokine TNF- $\alpha$ inhibits $\mathrm{Na}(+)$-glutamine cotransport in intestinal epithelial cells. Can J Physiol Pharmacol 91: 275-284, 2013.

9. Gao X, Wang W, Wei S and Li W: Review of pharmacological effects of Glycyrrhiza radix and its bioactive compounds. Zhongguo Zhong Yao Za Zhi 34: 2695-2700, 2009 (In Chinese).

10. Asl MN and Hosseinzadeh $\mathrm{H}$ : Review of pharmacological effects of Glycyrrhiza sp. and its bioactive compounds. Phytother Res 22: 709-724, 2008.

11. Isbrucker RA and Burdock GA: Risk and safety assessment on the consumption of Licorice root (Glycyrrhiza sp.), its extract and powder as a food ingredient, with emphasis on the pharmacology and toxicology of glycyrrhizin. Regul Toxicol Pharmacol 46: $167-192,2006$

12. Shin EM, Zhou HY, Guo LY, Kim JA, Lee SH, Merfort I, Kang SS, Kim HS, Kim S and Kim YS: Anti-inflammatory effects of glycyrol isolated from Glycyrrhiza uralensis in LPS-stimulated RAW264.7 macrophages. Int Immunopharmacol 8: 1524-1532, 2008.

13. Franceschelli S, Pesce M, Vinciguerra I, Ferrone A, Riccioni G, Patruno A, Grilli A, Felaco M and Speranza L: Licocalchone-C extracted from Glycyrrhiza glabra inhibits lipopolysaccharide-interferon- $\gamma$ inflammation by improving antioxidant conditions and regulating inducible nitric oxide synthase expression. Molecules 16: 5720-5734, 2011.

14. Sun D, Zhou M, Ying X, Cheng B, Han Y, Nie Y, Hou Y and Bai G: Identification of nuclear factor-kappaB inhibitors in the folk herb Rhizoma Menispermi via bioactivity-based ultra-performance liquid chromatography/quadrupole time-of-flight mass spectrometry analysis. BMC Complement Altern Med 14: 356, 2014.

15. Mi Wi S, Park J, Shim JH, Chun E and Lee KY: Ubiquitination of ECSIT is crucial for the activation of p65/p50 NF-kappaBs in Toll-like receptor 4 signaling. Mol Biol Cell 26: 151-160, 2015.

16. Chen R, Li M, Zhang Y, Zhou Q and Shu HB: The E3 ubiquitin ligase MARCH 8 negatively regulates IL- $1 \beta$-induced $N F-\kappa B$ activation by targeting the IL1RAP coreceptor for ubiquitination and degradation. Proc Natl Acad Sci USA 109: 14128-14133, 2012.

17. Yin H, Liu Z, Li F, Ni M, Wang B, Qiao Y, Xu X, Zhang M, Zhang J, Lu H and Zhang Y: Ginsenoside-Rg1 enhances angiogenesis and ameliorates ventricular remodeling in a rat model of myocardial infarction. J Mol Med (Berl) 89: 363-375, 2011.
18. Park KR, Nam D, Yun HM, Lee SG, Jang HJ, Sethi G, Cho SK and Ahn KS: $\beta$-Caryophyllene oxide inhibits growth and induces apoptosis through the suppression of PI3K/AKT/mTOR/S6K1 pathways and ROS-mediated MAPKs activation. Cancer Lett 312: 178-188, 2011.

19. Kim CK, Choi YK, Lee H, Ha KS, Won MH, Kwon YG and Kim YM: The farnesyltransferase inhibitor LB42708 suppresses vascular endothelial growth factor-induced angiogenesis by inhibiting ras-dependent mitogen-activated protein kinase and phosphatidylinositol 3-kinase/Akt signal pathways. Mol Pharmacol 78: 142-150, 2010.

20. Institute of Laboratory Animal Resources (US). Committee on Care, Use of Laboratory Animals, and National Institutes of Health (US). Division of Research Resources: Guide for the care and use of laboratory animals. 8th edition. National Academies Press, Washington, DC, 2011.

21. Wang ZH, Hsieh CH, Liu WH and Yin MC: Glycyrrhizic acid attenuated glycative stress in kidney of diabetic mice through enhancing glyoxalase pathway. Mol Nutr Food Res 58: 1426-1435, 2014.

22. Khan R, Khan AQ, Lateef A, Rehman MU, Tahir M, Ali F, Hamiza OO and Sultana S: Glycyrrhizic acid suppresses the development of precancerous lesions via regulating the hyperproliferation, inflammation, angiogenesis and apoptosis in the colon of Wistar rats. PLoS One 8: e56020, 2013.

23. Wang ZX, Huang CY, Hua YP, Huang WQ, Deng LH and Liu KX: Dexmedetomidine reduces intestinal and hepatic injury after hepatectomy with inflow occlusion under general anaesthesia: A randomized controlled trial. Br J Anaesth 112: 1055-1064, 2014.

24. Livak KJ and Schmittgen TD: Analysis of relative gene expression data using real-tie quantitative PCR and the 2(-Delta Delta C(T)) Method. Methods 25: 402-408, 2001.

25. Best SM, Wolfinbarger JB and Bloom ME: Caspase activation is required for permissive replication of Aleutian mink disease parvovirus in vitro. Virology 292: 224-234, 2002.

26. Cipriani S, Mencarelli A, Chini MG, Distrutti E, Renga B, Bifulco G, Baldelli F, Donini A and Fiorucci S: The bile acid receptor GPBAR-1 (TGR5) modulates integrity of intestinal barrier and immune response to experimental colitis. PLoS One 6: e25637, 2011.

27. Reynolds JM, Martinez GJ, Nallaparaju KC, Chang SH, Wang YH and Dong C: Cutting edge: Regulation of intestinal inflammation and barrier function by IL-17C. J Immunol 189: 4226-4230, 2012.

28. Bhattacharjee S, Bhattacharjee A, Majumder S, Majumdar SB and Majumdar S: Glycyrrhizic acid suppresses Cox-2-mediated anti-inflammatory responses during Leishmania donovani infection. J Antimicrob Chemother 67: 1905-1914, 2012.

29. Orazizadeh M, Fakhredini F, Mansouri E and Khorsandi L: Effect of glycyrrhizic acid on titanium dioxide nanoparticles-induced hepatotoxicity in rats. Chem Biol Interact 220: 214-221, 2014.

30. Lord JD, Chen J and Kozarek RA: A case of fatal idiopathic enteritis and multiple opportunistic infections associated with dendritic cell deficiencies. J Gastrointestin Liver Dis 22: 87-91, 2013.

31. Miner P, Wedel M, Bane B and Bradley J: An enema formulation of alicaforsen, an antisense inhibitor of intercellular adhesion molecule-1, in the treatment of chronic, unremitting pouchitis. Aliment Pharmacol Ther 19: 281-286, 2004.

32. Huang CF, Wu TC, Wu CC, Lee CC, Lo WT, Hwang KS, Hsu ML and Peng HJ: Sublingual vaccination with sonicated Salmonella proteins and mucosal adjuvant induces mucosal and systemic immunity and protects mice from lethal enteritis. APMIS 119: 468-478, 2011.

33. Feng L, Zhu MM, Zhang MH, Wang RS, Tan XB, Song J, Ding SM, Jia XB and Hu SY: Protection of glycyrrhizic acid against AGEs-induced endothelial dysfunction through inhibiting RAGE/NF- $\kappa \mathrm{B}$ pathway activation in human umbilical vein endothelial cells. J Ethnopharmacol 148: 27-36, 2013.

34. Wu Q, Tang Y, Zhang J, Hu X, Wang Q and Huang J: Therapeutic effects of glycyrrhizic acid on asthma airway inflammation in mice and its mechanism. Zhonghua Yi Xue Za Zhi 94: 3338-3344, 2014 (In Chinese).

35. Jorge E, Vergara P and Martin MT: Ileal inducible nitric oxide synthase mRNA expression in response to stress is modified in Sprague-Dawley rats exposed to a previous intestinal inflammation. Stress 15: 62-73, 2012. 
36. Li J, Feng G, Liu J, Rong R, Luo F, Guo L, Zhu T, Wang G and Chu Y: Renal cell carcinoma may evade the immune system by converting $\mathrm{CD}^{+}{ }^{+}$Foxp $3^{-} \mathrm{T}$ cells into $\mathrm{CD}^{+}{ }^{+} \mathrm{CD} 25^{+} \mathrm{Foxp}^{+}$regulatory T cells: Role of tumor COX-2-derived PGE2. Mol Med Rep 3: 959-963, 2010.

37. Sun L, Liu J, Cui D, Li J, Yu Y, Ma L and Hu L: Anti-inflammatory function of Withangulatin A by targeted inhibiting COX-2 expression via MAPK and NF-kappaB pathways. J Cell Biochem 109: 532-541, 2010

38. Cherng JM, Tsai KD, Yu YW and Lin JC: Molecular mechanisms underlying chemopreventive activities of glycyrrhizic acid against UVB-radiation-induced carcinogenesis in SKH-1 hairless mouse epidermis. Radiat Res 176: 177-186, 2011.

39. Wang Y, Fu Y, Xue S, Ai A, Chen H, Lyu Q and Kuang Y: The M2 polarization of macrophage induced by fractalkine in the endometriotic milieu enhances invasiveness of endometrial stromal cells. Int J Clin Exp Pathol 7: 194-203, 2014.
40. Zhang X, Li C, Li J, Xu Y, Guan S and Zhao M: Protective effects of protocatechuic acid on acute lung injury induced by lipopolysaccharide in mice via p38MAPK and NF- $\kappa \mathrm{B}$ signal pathways. Int Immunopharmacol 26: 229-236, 2015.

41. Bredeson S, Papaconstantinou J, Deford JH, Kechichian T, Syed A, Saade GR and Menon R: HMGB1 promotes a p38MAPK associated non-infectious inflammatory response pathway in human fetal membranes. PLoS One 9: e113799, 2014.

42. Akiho H, Tokita Y, Nakamura K, Satoh K, Nishiyama M, Tsuchiya N, Tsuchiya K, Ohbuchi K, Iwakura Y, Ihara E, et al: Involvement of interleukin-17A-induced hypercontractility of intestinal smooth muscle cells in persistent gut motor dysfunction. PLoS One 9: e92960, 2014.

43. Wojcik M, Zieleniak A, Zurawska-Klis M, Cypryk K and Wozniak LA: Increased expression of immune-related genes in leukocytes of patients with diagnosed gestational diabetes mellitus (GDM). Exp Biol Med (Maywood), 2015. 